\title{
Proteomics and Transcriptomics Analysis Reveals Clues into the Mechanism of the Beneficial Effect of Electrical Stimulation on Rat Denervated Gastrocnemius Muscle
}

\author{
Gaoyan La ${ }^{a}$ Mouwang Zhou Jae-Young Lim ${ }^{\mathrm{b}}$ Seung-Lyul Oh \\ Huayi Xing ${ }^{a} \quad$ Nan Liu ${ }^{a}$ Yanyan Yang ${ }^{a}$ Xiaoxie Liu ${ }^{a} \quad L_{i j u n} Z_{h o n g}$ \\ aDepartment of Rehabilitation Medicine, Peking University Third Hospital, Beijing, China, ${ }^{\mathrm{b}}$ Aging \& \\ Mobility Biophysics Lab, Department of Rehabilitation Medicine, Seoul National University Bundang \\ Hospital, Seongnam-si, Republic of Korea, 'Medical and Health Analytical Center, Peking University \\ Health Science Center, Beijing, China
}

\section{Key Words}

Denervation - Electrical stimulation - Gastrocnemius muscle - Proteomics - Skeletal muscle function modules $•$ Transcriptomics

\begin{abstract}
Background/Aims: The aim of this study was to confirm the beneficial effects of electrical stimulation on denervated skeletal muscle and explore a novel underlying mechanism. Methods: Morphological and contractile analyses were performed on rats allocated to three groups: sham operation (SHAM), denervated (DN), and denervated and electrical stimulation (DN-SM). Proteomics, transcriptomics, bioinformatics, and skeletal muscle functional modules analysis were conducted to determine the changes in molecular expression resulting from electrical stimulation. Results: Rats in the DN-SM group maintained a greater muscle mass, muscle fiber diameter, and contractile properties than those of the DN group. A total of 66 proteins and 402 mRNAs were differentially expressed between groups. Bioinformatics analysis suggested that the $\mathrm{FoxO}$ and $\mathrm{p} 53$ signaling pathways play significant roles in structural protection. Skeletal muscle function modules analysis suggested that anti-apoptosis proteins (KCNA7, KCNJ11), muscle fiber type related proteins (TNNI1, TNNT1, ACTN2, MYOZ2, MYLK2, and MYOM2), M-line structural protein (MYOM2), dystrophin combined with glycoprotein complex proteins (SGCB, SGCD, and DTNA), and anti-fibrosis-related proteins (POSTN, COL1A1, COL1A2, COL6A1, COL6A2, COL6A3, FN1, and LUM), may be related to the effects of electrical stimulation. Conclusion: Electrical stimulation can maintain the denervated muscle morphology and function. Anti-apoptosis, inhibition of muscle fiber type differentiation,
\end{abstract}


protection against dystrophin-associated-glycoprotein complex mutation, and anti-fibrosis are potential mechanisms of the beneficial effects of electrical stimulation.

(C) 2019 The Author(s). Published by Cell Physiol Biochem Press GmbH\&Co. KG

\section{Introduction}

Skeletal muscle denervation is a common disease caused by trauma, inflammation, or a surgical procedure. Denervation of the skeletal muscle was reported to be associated with structural changes such as atrophy [1], sarcomeric disorganization, myofibrillar disruption, changes in the number of ribosomes, changes in the number and size of mitochondria, and changes in sarcoplasmic reticulum morphology [2-4]. In addition, physiological disorders have been associated with denervated skeletal muscle, including delay of the contraction speed [5], reduction of developed tension [6], increase in the threshold for impulse generation [7], alteration in resting membrane potential [8], and changes in the muscle activation process [9].

Electrical stimulation has been clinically used as a treatment to relieve the muscle mass loss in a denervated skeletal muscle [10]. The most important positive effects of electrical stimulation on denervated skeletal muscle are the maintenance of muscle size and improvement in the contractile properties [11-14]. Despite extensive effort, the underlying mechanism mediating these beneficial effects remains unclear. To date, electrical stimulation has been shown to exert anti-apoptosis effects by reducing the upregulation of BAX, BCL-2 [15], and caspase-12 [16] expression, and by promoting the proliferation and differentiation of satellite cells $[14,17]$. Moreover, electrical stimulation was shown to regulate the abnormal expression and destabilization of acetylcholine receptor caused by denervation [18], reorganize the excitation-contraction coupling apparatus [19], and change the muscle fiber composition [20]. However, these previous studies generally focused on a single hypothesized mechanism, and there has been no large-scale analysis to uncover the mechanism of the beneficial effects of electrical stimulation on denervated muscle.

Proteomics and transcriptomics approaches enable conducting relatively comprehensive global analyses. Label-free quantitative proteomics combined with liquid chromatographytandem mass spectrometry (LC-MS/MS) is a reliable, versatile, and cost-effective method [21] that can effectively measure the abundance of proteins and peptides in different biological conditions by assaying thousands of proteins from complex biological samples simultaneously. Several proteomics studies have been conducted to analyze the proteins expressed in denervated skeletal muscle, revealing the activation of several biomarkers and pathways that might contribute to denervation-induced muscle atrophy [22, 23]; however, the influence of electrical stimulation on skeletal muscle denervation has not yet been evaluated with proteomics or transcriptomics approaches.

Therefore, in the present study, we analyzed the effects of electrical stimulation on the morphology and contractile properties of the denervated skeletal muscle in rats, and then conducted proteomics and transcriptomics analysis to determine the changes in muscle proteins with and without electrical stimulation. To determine the functional mechanism of the identified proteins and mRNA, bioinformatics analysis was carried out using Gene Ontology (GO) terms and Kyoto Encyclopedia of Genes and Genomes (KEGG) pathway analysis to detect the probable targets. Moreover, proteins that were differentially expressed after denervation and electrical stimulation were mapped to reported muscle function modules. Overall, these results should contribute new insight into the molecular mechanism by which electrical stimulation can maintain the muscle-specific physiological properties and suggest specific targets and pathways for the development of novel treatments that have the same beneficial effects. 


\section{Cellular Physiology Cell Physiol Biochem 2019;52:769-786 \begin{tabular}{ll|l} 
and Biochemistry & DOI: $10.33594 / 000000054$ & 2019 The Author(s). Published by \\
Published online: 2 April 2019 & \begin{tabular}{l} 
Cell Physiol Biochem Press GmbH\&Co. KG \\
\hline
\end{tabular}
\end{tabular} \\ La et al.: A Study of Electrical Stimulation on Denervated Muscle}

\section{Materials and Methods}

\section{Animals}

Sixty male 8-week-old Sprague-Dawley rats (Department of Laboratory Animal Science in Peking University Health Science Center, Beijing, China) were used in this study. The rats were housed in a temperature-controlled room at $20^{\circ} \mathrm{C}$, with alternating 12:12 light/darkness cycles, and diet and water provided ad libitum. All animals were randomly assigned to one of the following three groups ( $\mathrm{n}=20$ per group): sham-operated controls (SHAM), sciatic nerve denervation group (DN), and sciatic nerve denervation and daily electrical stimulation for 28 days (DN-SM). This study was approved by the Committee on the Ethics of Animal Experiments of Peking University (permit number: LA201456). All procedures were conducted in strict accordance with the recommendations of the Chinese Laboratory Animal Requirements of Environment and Housing Facilities. All animals survived until sacrifice.

\section{Nerve injury procedures}

The rats were anesthetized with intraperitoneal sodium pentobarbital $(6 \mathrm{mg} / 100 \mathrm{~g})$ and shaved before exposing the left sciatic nerve trunk. In the DN and DN-SM groups, the area approximately $1 \mathrm{~cm}$ proximal to the sciatic bifurcation was crushed with a hemostatic forceps for $30 \mathrm{~s}$. The rats in the SHAM group underwent the same procedure but without injury to the nerve.

\section{Electrical stimulation}

Electrical stimulation was conducted according to a previously described method [16]. In brief, 15 min after anesthesia by sodium pentobarbital, two surface electrodes were placed on the skin: one placed on one side near the Achilles tendon and the other placed at the opposite side near the popliteal fossa. The stimulator (ZS Dichuang, China) connected to the electrodes was set to a constant voltage of $25 \mathrm{~V}$, current of $1 \mathrm{~mA}$, pulse rate of $2 \mathrm{~Hz}$, and pulse width of $300 \mathrm{~ms}$, and was applied 10 min per day.

\section{Muscle sample preparation}

Rats were sacrificed and the left gastrocnemius muscles were rapidly removed. The wet weight and original length of the gastrocnemius muscle were measured in preparation of contractile measurements $(\mathrm{n}=$ 8 per group) and electron microscope analyses ( $n=3$ per group). Six gastrocnemius muscles per group were cut into four parts and used for single-muscle fiber measurement, H\&E staining, immunohistochemistry, and western blot analysis. The remaining samples ( $\mathrm{n}=3$ per group) were cut and quickly placed in liquid nitrogen for at least $15 \mathrm{~min}$, and then stored at $-80^{\circ} \mathrm{C}$ until LC-MS/MS and RNA-Seq analysis, which was conducted within one month.

\section{H\&E staining and immunohistochemistry}

Gastrocnemius muscle samples were fixed in $10 \%$ formalin for $24 \mathrm{~h}$, paraffin-embedded, and a microtome (Leica, Germany) was used to prepare 5 - $\mu \mathrm{m}$-thick sections. After drying overnight at $40^{\circ} \mathrm{C}$, the sections were stained with H\&E using a Leica autostainer xl (Leica, Germany).

For immunohistochemistry, the slides were immersed in xylene three times for $15 \mathrm{~min}$ each, anhydrous ethanol twice for $5 \mathrm{~min}$ each, $95 \%$ ethanol twice for $5 \mathrm{~min}$ each, and $80 \%$ ethanol twice for $5 \mathrm{~min}$ each, and then incubated in $3 \%$ methanol $-\mathrm{H}_{2} \mathrm{O}_{2}$ for $10 \mathrm{~min}$. The slides were then incubated with the primary antibody Anti-Fast (1:1200, ab51263, Abcam, Cambridge, UK). After overnight incubation at $4{ }^{\circ} \mathrm{C}$, the slides was washed in PBS three times for 5 min each, reacted with the secondary antibody (goat anti-mouse, PV6002, Zhongshan, China) for $30 \mathrm{~min}$, and then washed again with PBS three times for 5 min each, and finally stained with DAB for $10 \mathrm{~s}$.

The samples were scanned by a NanoZommer scanner (Humamatsu, Sydney, Australia). A transparent paper containing a $5 \times 10$ grid with squares of equal sizes was then placed over the image. All squares were numbered from 1-50, and a random number table was used to choose at least five squares for analysis of the area and diameter of each skeletal muscle fiber in the square so that at least 50 muscle fibers were analyzed per sample. The area and diameter of the muscle fiber was measured with image analysis software (NDP, View 2). 


\section{Cellular Physiology Cell Physiol Biochem 2019;52:769-786 \\ \begin{tabular}{ll|l} 
and Biochemistry $10.33594 / 000000054$ & $\begin{array}{l}\text { Do } 2019 \text { The Author(s). Published by } \\
\text { Published online: } 2 \text { April } 2019\end{array}$ & $\begin{array}{l}\text { Cell Physiol Biochem Press GmbH\&Co. KG } \\
\text { and }\end{array}$
\end{tabular}}

La et al.: A Study of Electrical Stimulation on Denervated Muscle

Ultrastructure analysis of muscle fibers

To observe the ultrastructure of the muscle, the samples were cut into longitudinal fragments and fixed in $2.5 \%$ glutaraldehyde at $4^{\circ} \mathrm{C}$. The samples were then washed in $0.1 \mathrm{~mol} / \mathrm{L}$ PBS, and post-fixed in $1 \%$ osmium tetroxide for $2 \mathrm{~h}$. The samples were then dehydrated in a graded series of alcohol and acetone solutions (50\% ethanol, $70 \%$ ethanol, $80 \%$ acetone, $90 \%$ acetone, $100 \%$ acetone $\mathrm{v} / \mathrm{v}$ ) for $10 \mathrm{~min}$ each, and embedded in epoxy resin overnight and polymerized at $35^{\circ} \mathrm{C}, 45^{\circ} \mathrm{C}$, and $60^{\circ} \mathrm{C}$ for $24 \mathrm{~h}$ each to obtain blocks. Ultrathin sections $(70 \mathrm{~nm})$ were cut with an ultramicrotome (LKB, Leica, Germany), which were stained with a saturated solution of uranyl acetate and lead citrate. The material was then examined and photographed under an electron microscope (JEM-1200EX, Japan).

\section{Contractile properties of the whole muscle}

Muscle contractile properties were measured as described previously [14, 24]. In brief, the excised fresh muscles were attached to the stimulating electrodes and bathed in Krebs-Ringer-Bicarbonate buffer at $37^{\circ} \mathrm{C}$ for $10 \mathrm{~min}$ with oxygen bubbled. Force production was measured by maximally stimulating the muscle for 20 stimulation bouts (voltage, $30 \mathrm{~V}$; training rate 2/min; training duration, $10 \mathrm{~s}$; pulse rate, 100 $\mathrm{Hz}$; duration, $0.1 \mathrm{~ms}$ ). A force transducer (ZS Dichuang, China) and the BL-420S software system (Taimeng, China) were used to monitor muscle contractions and capture digital signals, respectively. The peak force and the area under the curve were recorded and normalized to the cross-sectional area (CSA), calculated according to the following equation: CSA $\left(\mathrm{cm}^{2}\right)=$ muscle mass $(\mathrm{g}) /\left[\right.$ muscle length $\left.(\mathrm{cm}) \times 1.056 \mathrm{~g} / \mathrm{cm}^{3}\right]$.

\section{Single muscle fiber force measurements}

As described previously [25], three major solutions, including relaxing solution, skinning solution, and activating solution, were used for single muscle fiber force measurements in the present study. Muscle fiber bundles were homogenously picked up from the posterior of the gastrocnemius, which are mainly the fast-twitch fibers [26], incubated in cold relaxing solution, immersed into the skinning solution, and incubated for $24 \mathrm{~h}$ at $4^{\circ} \mathrm{C}$. Subsequently, the fibers were incubated in the following solutions at least for 30 min each: skinning solution containing $0.5 \mathrm{~mol} / \mathrm{L}, 1.0 \mathrm{~mol} / \mathrm{L}, 1.5 \mathrm{~mol} / \mathrm{L}$, and $2.0 \mathrm{~mol} / \mathrm{L}$ sucrose [27]. The muscle bundles were rapidly frozen in liquid nitrogen for more than $15 \mathrm{~min}$, and stored at $-80^{\circ} \mathrm{C}$ for less than three months.

Small muscle bundles were placed in 1\% Triton X-100 relaxing solution for 20 min. Individual fibers were dissected in relaxing solution and mounted on the test system (1400A, Aurora Scientific Inc., Canada) and fixed by T-clips. The sarcomere of single muscle fibers was set at $2.6 \mu \mathrm{m}$ and the single muscle fiber was regarded as a cylinder. An inverted microscope (Olympus, Japan) was used to observe the muscle fibers. The fibers were transferred from the relaxing solution to the activating solution, and the contraction of the fiber was observed. The peak force was calculated by the transducer and the normalized peak force was calculated dividing by the cross-sectional area.

\section{Protein preparation, $L C-M S / M S$, and label-free quantification}

The proteomics analysis was conducted as described previously [28]. Protein samples (200 mg) were digested according to the instructions for filter-aided sample preparation (FASP) [29]. High-pH reversedphase chromatography was then performed using a Dionex Ultimate 3000 Micro Binary HPLC pump system to separate the peptides. The eluted peptides were pooled into 15 fractions and vacuum-dried, and subjected to nano-electrospray ionization-LC-MS/MS analysis. The analysis was performed in triplicate on a nano-flow HPLC system (Easy-nLCII, Thermo Fisher Scientific, USA) connected to an LTQ-Orbitrap Velos Pro (linear quadrupole ion trap-orbitrap mass analyzer) mass spectrometer equipped with a Nanospray Flex Ion Source (Thermo Fisher Scientific, Waltham, MA, USA). In brief, the peptides were separated in a pre-column (Easy-column C18-A1, $100 \mu \mathrm{m}$ I.D. $\times 20 \mathrm{~mm}, 5 \mu \mathrm{m}$ ) and a reversed-phase C18 column (Easycolumn C18-A2, $75 \mu \mathrm{m}$ I.D. $\times 100 \mathrm{~mm}, 3 \mu \mathrm{m}$ ). The eluting peptides were ionized with an electrospray maintained at $2.2 \mathrm{kV}$, and the 15 most abundant ions detected in the full-MS scan were measured in the LTQ part by collision-induced dissociation. Each group was analyzed in three biological replicates. The raw data analysis was conducted with MaxQuant software (version1.4.1.2, http://www.maxquant.org/). For protein identification, the MS/MS data were submitted to the Uniprot human protein database (release 3.43, 72, 340 sequences). Label-free quantization (LFQ) was also performed in MaxQuant. The minimum ratio count for LFQ was set to 2 , and the match-between-runs option was enabled; the other parameters 


\section{Cellular Physiology Cell Physiol Biochem 2019;52:769-786

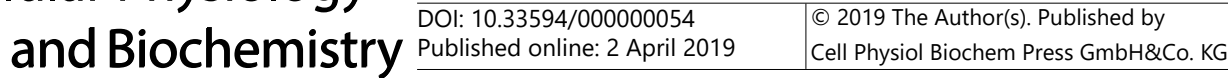

La et al.: A Study of Electrical Stimulation on Denervated Muscle

were set to the default. The up-regulated and down-regulated proteins were defined as those with $\mathrm{p}<0.05$. When a protein was up-regulated in the DN/SHAM and down-regulated in the DN-SM/DN group or vice versa, it was regarded as differentially expressed after denervation and electrical stimulation compared to after denervation only.

\section{RNA extracting and sequencing}

RNA preparation, library construction, and sequencing were carried out by the Beijing Genomics Institute (Beijing, China) using their high-throughput genome sequencing platform, BGISEQ-500. Briefly, flash frozen muscle samples were homogenized in TRIzol (Thermo Fisher Scientific) and total RNA generated according to protocol. Qualitative and quantitative analysis of the RNA was conducted using a Qubit Fluorometer (Thermo Fisher Scientific), an Agilent 2100 bioanalyzer (Agilent Technologies, Palo Alto, CA, USA), a NanoDrop 2000 microvolume spectrophotometer (Thermo Fisher Scientific), and a microplate reader (Thermo Fisher Scientific). Following DNase I digestion, mRNA isolation, mRNA fragmentation, and cDNA synthesis and repair, a total RNA library was prepared and validated on the Agilent 2100 bioanalyzer. Expression levels of mRNA were assessed using BGISEQ-500, according to the manufacturer's instructions. After filtering, clean reads were mapped to references using the HISAT and Bowtie2 tools. The system's quality control requires a gene unique mapping ratio higher than $80 \%$ and a genome mapping ratio higher than 50\%. Differentially expressed genes were determined by $\mathrm{P}$ value $<0.05$. If a gene was up-regulated in the DN/SHAM and down regulated in the DN-SM/DN group or vice versa, it was regarded as differentially expressed gene.

GO and KEGG pathway analysis

Analysis of up- and down-regulated genes was performed as described previously [28]' and subjected to hierarchical clustering analysis of GO terms and KEGG pathways with OmicsBean (http://www.omicsbean. com) [30].

\section{Muscle functional modules analysis}

Mukund et al. [31] developed a list of muscle function modules (Supplementary Table 1 - all supplementary material available online at www.cellphysiolbiochem.com) according to previous work [32], including a total of 23 functional modules such as neuromuscular junction, excitation contraction coupling, contraction, cytoskeleton, extracellular matrix, mitochondrial energy metabolism, hypertrophy, atrophy, inflammation, regulators, fiber type maintenance, vasculogenesis, and oxidative stress modules. Determination of the most relevant biomarkers in the skeletal muscle modules would facilitate further studies to explore the underlying mechanism of skeletal muscle-related diseases and treatments. We mapped the proteins in the DN-SM group to the skeletal muscle functional modules lists. To identify the functional proteins most closely related to anti-atrophy and contractility, we mainly focused on proteins in the neuromuscular junction, excitation contraction coupling, contraction, cytoskeleton, and extracellular matrix modules, and selected the most relevant proteins based on a review of the related literature.

\section{Proteomic validation by western blotting}

To validate the findings of proteomics analysis, western blotting was performed for selected proteins as positive and negative controls. We randomly selected MFF and ATP8A1 as positive controls and MBP as a negative control. Proteins extracted from the muscle samples ( $\mathrm{n}=3$ per group) were lysed in RIPA buffer (Applygen, Beijing, China) containing protease inhibitor cocktail (Roche, Basel, Switzerland) for 30 min on ice, and the protein concentration was determined using a bicinchoninic acid assay (Applygen, Beijing, China). After the addition of loading buffer (Applygen, Beijing, China), the proteins were electrophoresed on $12 \%$ SDS-PAGE gels, transferred to nitrocellulose membranes (Applygen, Beijing, China), blocked with fresh blocking buffer [5\% non-fat milk in TBS with Tween 20 (TBST); Applygen, Beijing, China] at room temperature for $60 \mathrm{~min}$, and probed with primary antibodies against MFF (1:1000, Abcam, Cambridge, UK), ATP8A1 (1:1000, Abcam, Cambridge, UK), MBP (1:1000, Abcam, Cambridge, UK), and GAPDH (1:10000, Abcam, Cambridge, UK) at $4^{\circ} \mathrm{C}$ overnight. The membrane was washed three times for 10 min each using TBST (Applygen, Beijing, China), and then incubated with IRDye 800CW-conjugated goat anti-rabbit IgG or goat anti-mouse IgG (both at 1:10000 dilution) secondary antibody at room temperature for $1 \mathrm{~h}$, and 


\section{Cellular Physiology Cell Physiol Biochem 2019;52:769-786 \\ \begin{tabular}{ll|l} 
and Biochemisty $10.33594 / 000000054$ & (c) 2019 The Author(s). Published by \\
Cell Physiol Biochem Press GmbH\&Co. KG
\end{tabular}}

La et al.: A Study of Electrical Stimulation on Denervated Muscle

washed three more times in TBST buffer. The membrane was finally visualized with the Odyssey infrared imaging system (LI-COR Biosciences). GAPDH levels were used as loading controls.

Real-time PCR

A total of $2 \mu \mathrm{g}$ RNA from the remaining samples ( $\mathrm{n}=3$ per group) after RNA-seq was reverse-transcribed for cDNA using a First Strand cDNA synthesis Kit (Tiangen, Beijing, China) following the manufacturer's instructions. The real-time reactions were performed using SYBR Green PCR Master Mix (Tiangen, Beijing, China). All reactions were performed in triplicate. The relative gene expression levels were calculated using the $2^{-\Delta \Delta C \mathrm{~T}}$ method. Gapdh was used as the housekeeping gene. The primers are listed in Supplementary Table 2 .

Statistical analysis

Results are displayed as mean \pm SEM. Differences between two groups were analyzed using Student's t-test. A statistically significant difference was considered when $\mathrm{P}<0.05$.

Data availability

Mass spectrometry proteomics raw data have been deposited at iProX (http://www.iprox.org) with the dataset identifier IPX0001260001. The RNA-seq data are available in the GEO database and the reference series is GSE117440.

\section{Results}

Morphology of the gastrocnemius muscle

H\&E staining of transverse and longitudinal sections showed a significant decrease in the fiber CSA $(p<0.001)$ and diameter $(p<0.001)$ in the DN and DN-SM groups compared to those of the SHAM group. In addition, the DN-SM group had a larger CSA $(p<0.001)$ and diameter $(\mathrm{p}<0.001)$ than those of the DN group (Fig. 1A-H). Moreover, deep staining of the fast fibers (Fig. 1I) showed more obvious changes in muscle fiber area and diameter in fast fibers than in slow fibers (Fig. 1J, K, L).

Transmission electron micrographs of longitudinal sections of the rat gastrocnemius showed that in the DN group, some sarcomeres and Z-lines were misaligned or not detected, and the myofibrils appeared to be swollen with an enlarged sarcoplasmic reticulum (Fig. 2AI). The width of the Z line increased significantly in both the DN $(p<0.001)$ and DN-SM $(p<$ 0.001 ) groups, although this increase was more obvious in the DN group (Fig. 2J). Clear length differences of the sarcomeres were observed between the DN and DN-SM groups (Fig. 2K). Central nuclei were also apparent (Fig. 2B, E, H), and the nuclear shape in the SHAM group was similar to that observed in normal animals. However, shrinking of the central nuclei to different extents was observed in the other two groups, with greater shrinkage observed in the DN group than in the DN-SM group. The mitochondria had a normal appearance in the SHAM group but were swollen in the DN group (Fig. 2C, F, I).

\section{Contractile properties of the gastrocnemius muscle}

As shown in Table 1, a decrease in muscle wet weight was observed in the DN and DNSM groups compared with that of the SHAM group, and the wet weight was greater in the DN-SM group than that of the DN group. Peak force, normalized peak force (a measure of the strength of skeletal muscle contraction), area under the curve, and normalized area under the curve (a measure of the muscle strength and fatigue resistance) were calculated as the muscle contractile properties. As expected, these parameters were all greater in the SHAM group, were the lowest in the DN group, and those of the DN-SM group were intermediate. As shown in Table 2, the fiber diameter decreased by $26.61 \%$ and $22.79 \%$ in the DN group and DN-SM group, respectively, compared with that of the SHAM group. The CSA showed a similar trend to single muscle fiber diameter. The single muscle fiber length of the three groups ranged from $1.2 \mathrm{~cm}$ to $1.4 \mathrm{~cm}$. In the DN and DN-SM groups, the peak force was 


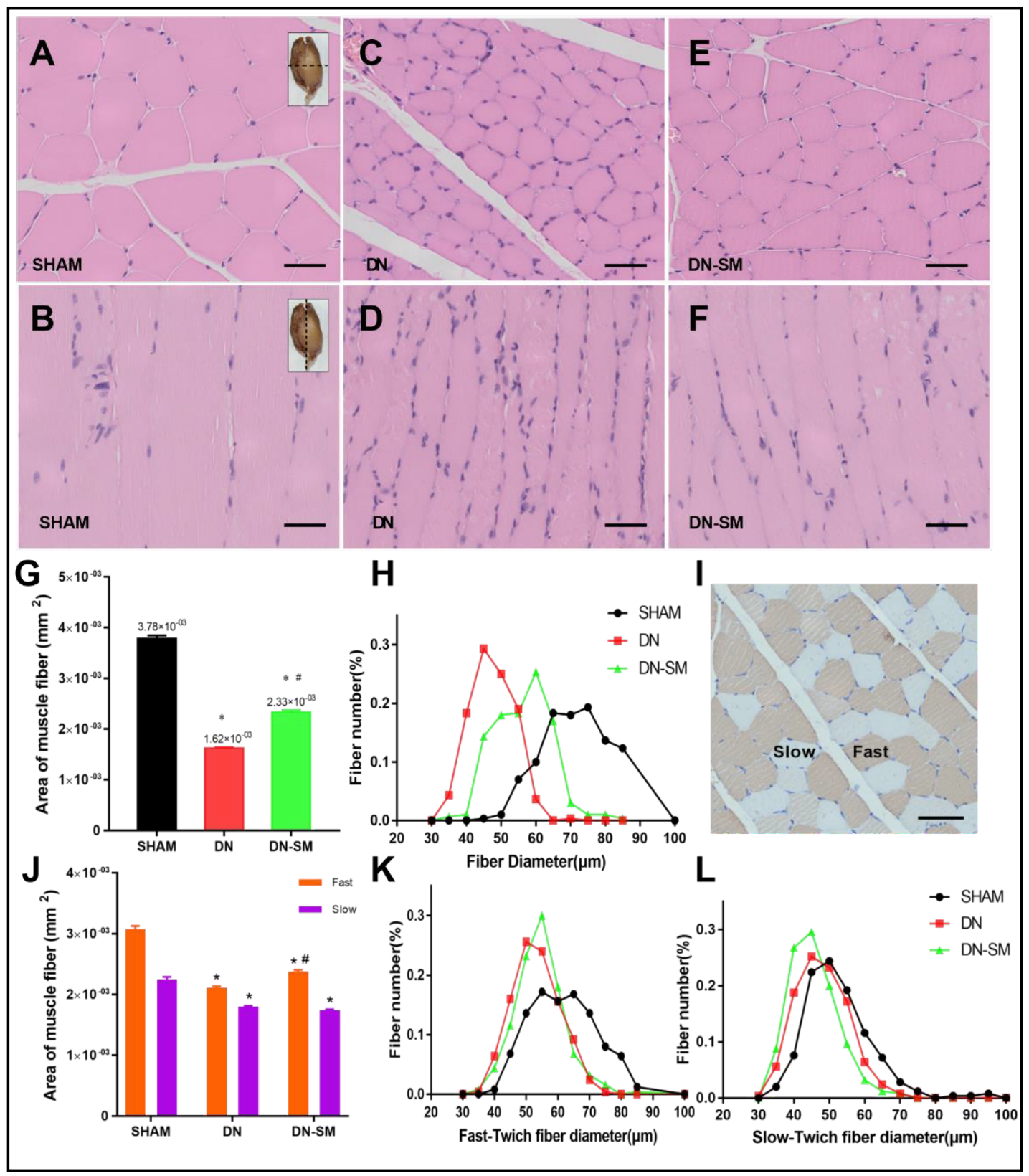

Fig. 1. Structure of the gastrocnemius muscle. (A-H) Representative H\&E staining light graph showing the muscle fiber area $(G)$ and diameter $(H)$ (Scale bar $=100 \mu \mathrm{m}, \mathrm{n}=6$ per group). (I-L) Immunohistochemistry results showing the fast- and slow-type muscle fiber area and diameter, respectively (Scale bar $=100 \mu \mathrm{m}$, $\mathrm{n}=6$ per group). Data are presented as mean \pm SEM. SHAM, sham operation; DN, denervation; DN-SM, denervation and electrical stimulation; ${ }^{*} \mathrm{P}<0.05$, significantly different from SHAM; ${ }^{\#} \mathrm{P}<0.05$, significantly different from DN.

$67.86 \%$ and $46.43 \%$ lower than that in the SHAM group, respectively. In addition, the peak force in the DN-SM group was greater than that in the DN group $(p<0.05)$. The normalized peak force was still the highest in the SHAM group and the lowest in the DN group, and there was no difference in the normalized peak force between the SHAM and DN-SM groups. The normalized peak force in the DN group was reduced by $41.57 \%$ and $39.57 \%$ compared to that in the SHAM and DN-SM group, respectively (Table 2). 


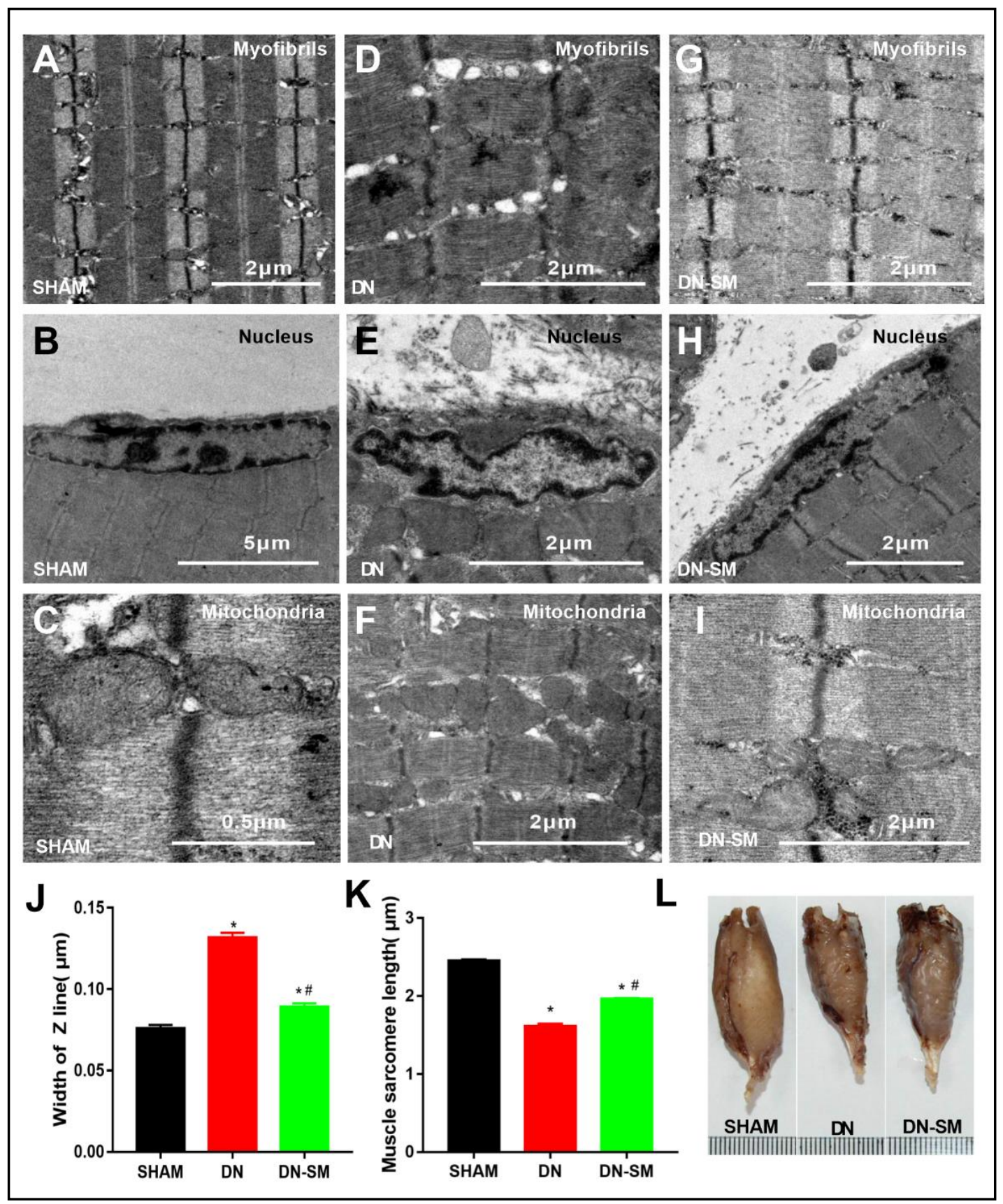

Fig. 2. Microstructure of gastrocnemius muscle fibers. (A-I) Transmission electron micrographs of longitudinal sections of the rat gastrocnemius muscle. Ultrastructure of skeletal myofibrils $(A, D, G)$, nucleus $(B, E, H)$, and mitochondria (C, F, I), width of the $\mathrm{Z}$ line (J), and the muscle sarcomere length (K) in the three groups $(n=3)$. (L) Representative images of the gastrocnemius muscle in the three groups.

\section{Differentially expressed proteins and $m R N A s$}

An intersection of 66 differentially expressed proteins (displayed in Supplementary Table 3) was identified from the two comparisons (DN vs SHAM and DN-SM vs DN; triplicate analysis, Fig. 3). Overall, 402 differentially expressed mRNAs (displayed in Supplementary Table 4) were identified from RNA-seq (DN vs SHAM and DN-SM vs DN; triplicate analysis). 


\section{Cellular Physiology Cell Physiol Biochem 2019;52:769-786 \begin{tabular}{ll|l} 
DOI: 10.33594/000000054 & (c) 2019 The Author(s). Published by \\
Cell Physiol Biochem Press GmbH\&Co. KG
\end{tabular}}

La et al.: A Study of Electrical Stimulation on Denervated Muscle

Table 1. Gastrocnemius muscle characteristic and contractile parameters. Data presented as mean \pm SEM. Sample size per group, $\mathrm{n}=20$ for Body weight- 1 and Body weight- $2, \mathrm{n}=8$ for all the other variables; SHAM, sham operation; DN, denervation; DN-SM, denervation and electrical stimulation; Body weight-1, body weight before injury; Body weight-2, body weight 4-week after injury; PCSA, physiological cross-sectional area; ${ }^{*} \mathrm{P}<0.05$ vs SHAM, ${ }^{\#} \mathrm{P}<0.05$ vs $\mathrm{DN}$

\begin{tabular}{lccc}
\hline Parameter & SHAM & DN & DN-SM \\
\hline Body weight-1 $(\mathrm{g})$ & $283.90 \pm 1.84$ & $288.20 \pm 2.81$ & $287.40 \pm 1.23$ \\
Body weight-2 $(\mathrm{g})$ & $420.15 \pm 5.30$ & $406.15 \pm 6.57$ & $402.25 \pm 3.62$ \\
Muscle wet weight $(\mathrm{g})$ & $2.95 \pm 0.03$ & $1.56 \pm 0.10^{*}$ & $2.02 \pm 0.07^{* \#}$ \\
Muscle wet weight/Body weight-2·10-3 & $7.02 \pm 0.11$ & $3.84 \pm 0.24^{*}$ & $5.02 \pm 0.20^{* \#}$ \\
Muscle length $(\mathrm{cm})$ & $3.86 \pm 0.02$ & $3.81 \pm 0.05$ & $3.88 \pm 0.04$ \\
PCSA $\left(\mathrm{cm}^{2}\right)$ & $0.72 \pm 0.01$ & $0.39 \pm 0.02^{*}$ & $0.49 \pm 0.02^{* \#}$ \\
Peak force(N) & $7.26 \pm 0.17$ & $2.06 \pm 0.12^{*}$ & $3.85 \pm 0.10^{* \#}$ \\
Normalized peak force $\left(\mathrm{N} / \mathrm{cm}^{2}\right)$ & $10.05 \pm 0.17$ & $5.39 \pm 0.32^{*}$ & $7.85 \pm 0.27^{* \#}$ \\
Area under the curve $(\mathrm{N} \cdot \mathrm{s})$ & $113.87 \pm 4.34$ & $29.55 \pm 2.16^{*}$ & $60.88 \pm 1.92^{* \#}$ \\
Normalized area under the curve $\left(\mathrm{N} \cdot \mathrm{s} / \mathrm{cm}^{2}\right)$ & $157.64 \pm 5.87$ & $76.78 \pm 4.26^{*}$ & $123.97 \pm 3.57^{* \#}$ \\
\hline
\end{tabular}

Table 2. Changes in single muscle fiber diameter, cross-sectional area, peak force, specific force, and maximal shortening velocity. Data presented as mean \pm SEM. The number of muscle fibers $n=20$ per group; SHAM, sham operation; DN, denervation; DN-SM, denervation and electrical stimulation; CSA $_{\mathrm{F}}$, cross-sectional area of single muscle fiber; ${ }^{*} \mathrm{P}<0.05$, significantly different from $\mathrm{SHAM}$; ${ }^{\#} \mathrm{P}<0.05$, significantly different from $\mathrm{DN}$

\begin{tabular}{lccc}
\hline Parameter & SHAM & DN & DN-SM \\
\hline Diameter $(\mu \mathrm{m})$ & $79.85 \pm 3.38$ & $58.60 \pm 1.87^{*}$ & $61.65 \pm 4.05^{*}$ \\
$\mathrm{CSA}_{\mathrm{F}}\left(\mu \mathrm{m}^{2}\right)$ & $5718.61 \pm 439.94$ & $2748.98 \pm 186.31^{*}$ & $3229.87 \pm 446.12^{*}$ \\
Fiber length $(\mathrm{cm})$ & $1.28 \pm 0.06$ & $1.27 \pm 0.07$ & $1.34 \pm 0.04$ \\
Peak force $(\mathrm{mN})$ & $0.28 \pm 0.02$ & $0.09 \pm 0.01^{*}$ & $0.15 \pm 0.01^{* \#}$ \\
Normalized peak force $\left(\mathrm{mN} / \mathrm{mm}^{2}\right)$ & $58.07 \pm 4.75$ & $33.93 \pm 2.91^{*}$ & $56.15 \pm 5.79 \#$ \\
\hline
\end{tabular}

\section{GO and KEGG pathway analysis}

The detailed GO and KEGG pathway analyses results for the 66 differentially expressed proteins and 402 differentially expressed mRNAs are displayed in Supplementary Tables 3 and 4. Briefly, differentially expressed proteins were enriched in intracellular and intracellular GO terms (Fig. 4A). The KEGG results of differentially expressed mRNAs revealed enrichment in the Fox0 signaling pathway (including Ccnb1, Ccnd1, Igf1, Mapk3, Cat, Prmt1, Prkag1, Rbl2, Usp7, $\mathrm{P}=0.00185$ ) and the p53 signaling pathway (including Rrm2, Ccnb1, Ccnd1, Tp53, Igf1, Cdk4, P= 0.00322) (Fig. 4B).

\section{Skeletal muscle functional modules analysis}

The skeletal muscle modules analysis method used in our study has been developed and adapted by many researchers. Functional modules represent significant units required for normal muscle activity, and provide a framework to simplify high-throughput analyses by mapping proteins to the module list. To further analyze the proteomics results in a more skeletal muscle-specific context, we mapped the proteins and mRNAs to previously identified skeletal muscle functional modules. As our main goal was to provide insight into the underlying mechanism of electrical stimulation for maintaining the muscle mass and contractile properties of denervated skeletal muscle, and the $\mathrm{GO}$ analysis revealed enrichment in intracellular terms, we focused on four modules: excitation-contraction coupling, muscle contraction, cytoskeleton, and extracellular matrix. A total of 19 proteins were selected after this analysis (Table 3). 
Cellular Physiology \begin{tabular}{ll|l} 
and Biochemistry & $\begin{array}{l}\text { DOl: 10.33594/000000054 } \\
\text { Published online: } 2 \text { April } 2019\end{array}$ & $\begin{array}{l}\text { O } 2019 \text { The Author(s). Published by } \\
\text { Cell Physiol Biochem Press GmbH\&Co. KG }\end{array}$ \\
\cline { 2 - 3 }
\end{tabular}

La et al.: A Study of Electrical Stimulation on Denervated Muscle

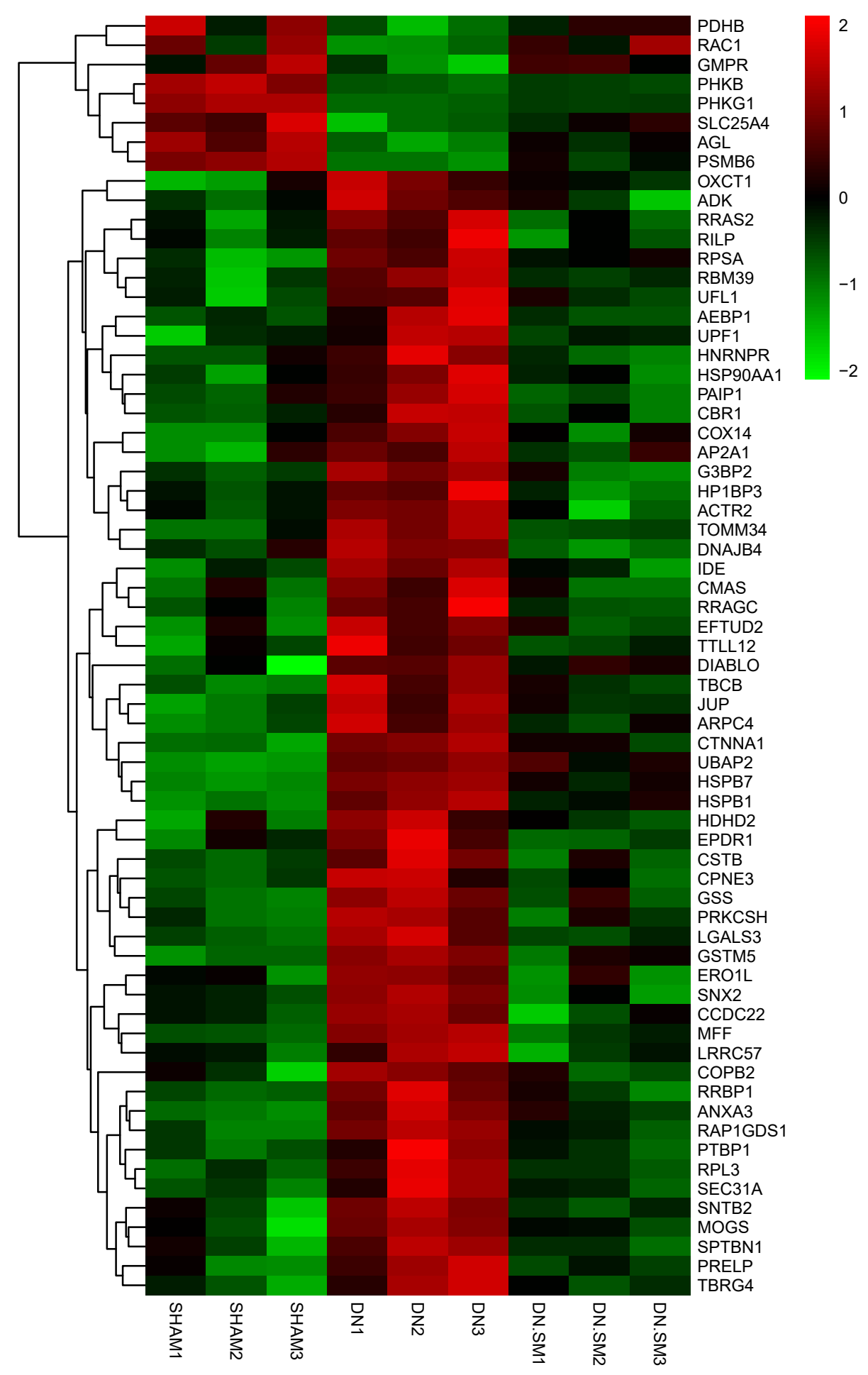

Fig. 3. Heat map of the 66 differentially expressed proteins identified from the LC-MS/MS results (DN vs SHAM, DN-SM vs DN). 


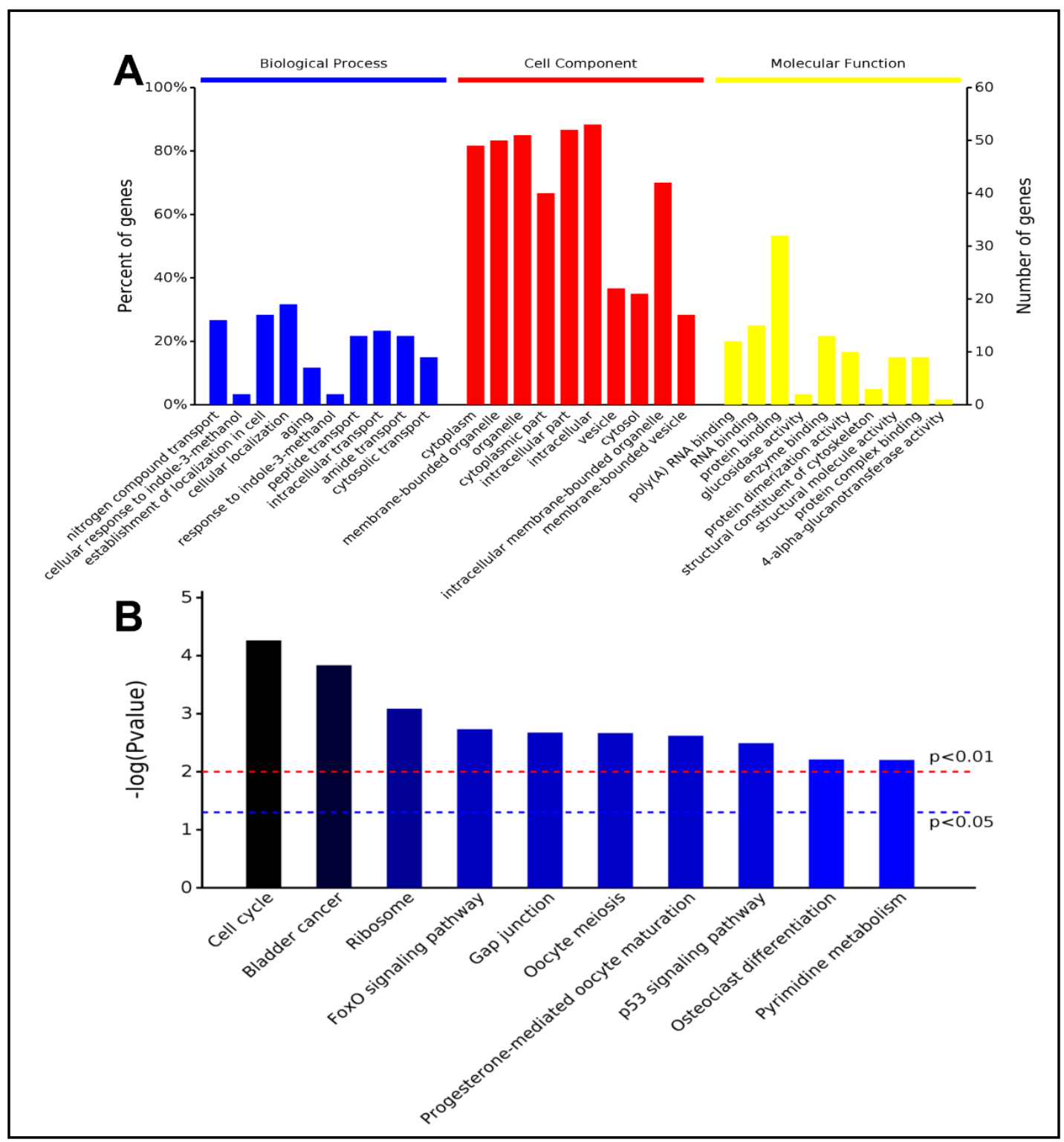

Fig. 4. Bioinformatics analysis of the differentially expressed proteins and mRNAs. (A) GO analysis of 66 differentially expressed proteins, (B) KEGG pathway analysis of 402 differentially expressed mRNAs.

\section{Confirmation of proteomics and transcriptomics analysis}

We used western blot to validate the protein expression level of MFF and ATP8A1 in the SHAM, DN, and DN-SM groups. As shown in Fig. 5, compared to the DN group, MFF expression was significantly down-regulated and ATP8A1 expression was significantly upregulated after electrical stimulation, which was consistent with the proteomics results (Supplementary Table 3). The negative control MBP expression in western blotting was also in line with the results of proteomics.

Six genes (Des, Rpl18a, Cyc1, Sparc, Lgals1, and Ndufb10) were randomly chosen for qPCR validation for the expression changes detected by RNA-seq (Supplementary Table 4). Both qPCRs and RNA-seq showed that the expression levels of these six genes were increased after denervation (DN vs SHAM) and decreased after treatment (DN-SM vs DN). The validation suggested good reliability of RNA-seq results (Fig. 5). 


\section{Discussion}

In this study, we confirmed the positive effect of electrical stimulation on rat denervated skeletal muscle with regards to both morphology and contractile function. Moreover, we conducted the proteomics and transcriptomics analysis on the muscles following denervation and electrical stimulation to gain insight into the mechanism underlying these beneficial effects. These results revealed some key molecular targets and candidate pathways that can provide a valuable research focus for future studies on the therapeutic mechanism of electrical stimulation.

The ability of electrical stimulation to maintain the muscle wet weight, diameter, and CSA of denervated muscle support previous studies using low-frequency electrical stimulation [11-14], although the stimulation parameters were slightly different. More obvious change of fast-type fibers following electrical stimulation is likely due to the fact that electrical stimulation-induced contractions are non-selective and could recruit fast motor units at a low force level [33]. The ultrastructure analysis suggested that electrical stimulation could maintain the sarcomere structure and prevent degeneration, which is consistent with previous reports [2,34].

The contractile apparatus used in our experiments was limited to the most important parameters both at the whole muscle and single-muscle level, indicating the contractile protective function of electrical stimulation in line with previous studies [14,35]; however, the present study confirmed these effects at both the whole muscle and single-muscle fiber level. In contrast to whole muscle contractile measurement, single-muscle fiber measurement can be used to measure the skinned single muscle fiber at the cellular level, thereby avoiding the potential influence of blood, fibrous membranes, collagen, and other factors [25]. Therefore, this measurement serves as a direct tool to assess the impairment or effect of sarcomeric proteins. In this study, the single-muscle fiber measurement demonstrated the beneficial effect of electrical stimulation on the contractile properties in a more specific way, thereby increasing the reliability of the results, and providing more specific evidence of the positive effect of electrical stimulation on denervation in the muscle.

The KEGG results revealed enrichment in the FoxO signaling pathway and the p53 signaling pathway. Interestingly, this is the first report of the effects of electrical stimulation

Table 3. List of selected proteins and mRNAs enriched in skeletal muscle function modules

\begin{tabular}{|c|c|c|c|c|c|}
\hline Gene names & Protein names & $\begin{array}{c}\text { Protein } \\
\text { (DN vs SHAM) }\end{array}$ & $\begin{array}{c}\text { Protein } \\
\text { (DN-SN vs DN) }\end{array}$ & $\begin{array}{c}\text { mRNA } \\
\text { (DN vs SHAM) }\end{array}$ & $\begin{array}{c}\text { mRNA } \\
\text { (DN-SM vs DN) }\end{array}$ \\
\hline \multicolumn{6}{|c|}{ Excitation-Contraction Coupling (ECC) } \\
\hline Kcnj11 & $\begin{array}{l}\text { ATP-sensitive inward rectifier } \\
\text { potassium channel } 11\end{array}$ & 0.37 & 1.53 & 0.58 & 1.18 \\
\hline Kcna7 & $\begin{array}{l}\text { Potassium voltage-gated channel } \\
\text { subfamily A member } 7\end{array}$ & 0.39 & 1.44 & 0.85 & 1.06 \\
\hline \multicolumn{6}{|c|}{ Muscle Contraction (MC) } \\
\hline Tnni1 & Troponin I, slow skeletal muscle & 5.39 & 0.37 & 2.02 & 0.62 \\
\hline Tnnt1 & Troponin T, slow skeletal muscle & 3.36 & 0.40 & 1.65 & 0.68 \\
\hline Actn2 & actinin alpha 2 & 2.83 & 0.26 & 1.42 & 0.73 \\
\hline Myoz2 & myozenin 2 & 9.86 & 0.18 & 1.28 & 0.79 \\
\hline Mylk2 & myosin light chain kinase 2 & 0.29 & 1.49 & 0.47 & 1.11 \\
\hline Myom2 & myomesin 2 & 0.46 & 1.14 & 0.91 & 1.42 \\
\hline \multicolumn{6}{|l|}{ Cytoskeleton } \\
\hline Sgcb & sarcoglycan, beta & 0.88 & 1.35 & 0.54 & 1.80 \\
\hline Sgcd & sarcoglycan, delta & 0.77 & 1.31 & 1.05 & 1.52 \\
\hline Dtna & dystrobrevin, alpha & 0.90 & 1.22 & - & - \\
\hline \multicolumn{6}{|c|}{ Extracellular Matrix (EMC) } \\
\hline Posth & periostin & 3.25 & 0.88 & 1.23 & 1.48 \\
\hline Col1a1 & collagen type I alpha 1 chain & 2.13 & 0.47 & 0.90 & 0.58 \\
\hline Col1a2 & collagen type I alpha 2 chain & 2.89 & 0.42 & 0.81 & 0.60 \\
\hline Col6a1 & collagen type VI alpha 1 chain & 1.65 & 0.64 & - & - \\
\hline Col6a2 & collagen type VI alpha 2 chain & 1.67 & 0.55 & 1.46 & 0.68 \\
\hline Col6a3 & collagen type VI alpha 3 chain & 1.42 & 0.66 & - & - \\
\hline Fn1 & fibronectin 1 & 2.92 & 0.47 & 0.96 & 0.68 \\
\hline Lum & lumican & 1.82 & 0.55 & 1.03 & 0.86 \\
\hline
\end{tabular}


in denervated muscle on Tp53, a tumor suppressor gene that plays an important role in cell proliferation and differentiation. NF-kB/ p53 is regulated by FoxO [36]. Tachtsis et al. reported an increase of Tp53 after acute endurance training in skeletal muscle [37]. Moreover, Tp53 plays an important role in maintaining mitochondrial content and function [38]. These results thus suggest new targets for investigating the underlying mechanism of electrical stimulation in denervated skeletal muscle.

The skeletal muscle functional modules analysis demonstrated that the regulation mechanism underlying the anti-atrophy and contractile effects of the muscle are mainly involved in four modules: excitation-contraction coupling, muscle contraction, cytoskeleton, and extracellular matrix, which are discussed in turn below.

Excitation-contraction coupling refers to the process from initial membrane depolarization through to action potential and activation of the Ca transient, and explains how myofilaments respond to the Ca transient to produce contractions. In this study, potassium voltage-gated channel subfamily J member 11 (KCNJ11), which is associated with this module, was found to be down-regulated after denervation and up-regulated after electrical stimulation. KCNJ11 is an ATP-sensitive $\mathrm{K}+$ channel, and has been reported to play a cardioprotective role during stress and to participate in apoptosis in cardiomyocytes [39]. However, its function in the skeletal muscle has not been studied, and our results suggest that it may be involved in the anti-apoptosis function of electrical stimulation. Potassium voltage-gated channel subfamily A member 7 (KCNA7) showed a similar changing pattern to KCNJ11, suggesting that it may also play a role in the regulatory mechanism of electrical stimulation treatment; however, this protein has not been studied widely and therefore warrants further attention.

The sarcomere is the basic unit of muscle contraction, where force generation is produced by the interaction between thick and thin filaments; thus, proteins related to the sarcomere are important for structural maintenance and contractile function. In the muscle contraction module, the expression levels of TNNI1, TNNT1, ACTN2, and MYOZ2 were all increased after denervation and decreased by electrical stimulation. TNNI1 and TNNT1 are the slow subunits of troponin, which is associated with the regulation of actomyosin and calcium-mediated interactions during contractions. In addition, the changes of different types of troponins after denervation depend on the muscle type and denervation period. In a fast-twitch muscle, 10 days after denervation, the slow-type troponin transcripts are upregulated, whereas in a slow-twitch muscle, the slow-type troponin is dramatically downregulated by skeletal muscle denervation as early as 4 days after nerve transection [40]. Actinin alpha 2 (ACTN2) expression is associated with improvement of muscular aerobic capacity and its levels are significantly higher in trained animals than in sedentary animals [41]. Myozenin 2, calsarcin-1 (MYOZ2) is specifically expressed in the adult cardiac muscle and slow-twitch skeletal muscle [42]. These proteins may be associated with the fiber type changes that occur after denervation and electrical stimulation, although one month might be too short for detecting the changes of fiber types; thus, changes of muscle fiber typespecific proteins might occur earlier than changes of the myosin isoforms. Our experiments were performed on the gastrocnemius muscle, a representative fast-twitch muscle, which has been shown to experience a fast-to-slow change during the early phase after denervation in rats [43]. As a pathological change, the fast-to-slow changes may be inhibited by electrical stimulation to some extent, which may explain our results.

Another two proteins that were up-regulated after denervation and down-regulated after electrical stimulation are myosin light chain kinase 2 kinase (MYLK2) and myomesin 2 (MYOM2). MYLK2 is highly expressed in the skeletal muscle, and is reported to be associated with the slow-to-fast transition in the soleus muscle [44]. MYOM2 is a major M-line structural protein, and its decrease was reported to cause significant delay in the speed of the cardiac muscle [45]. The M-line maintains the myosin myofilaments in order, links the contractile apparatus to the cytoskeleton for external force transfer, and localizes CK-based energy storage and transfer to the site of highest ATP demand [46]. Thus, MYOM2 may be a target protein of the contractile function changes caused by electrical stimulation. 


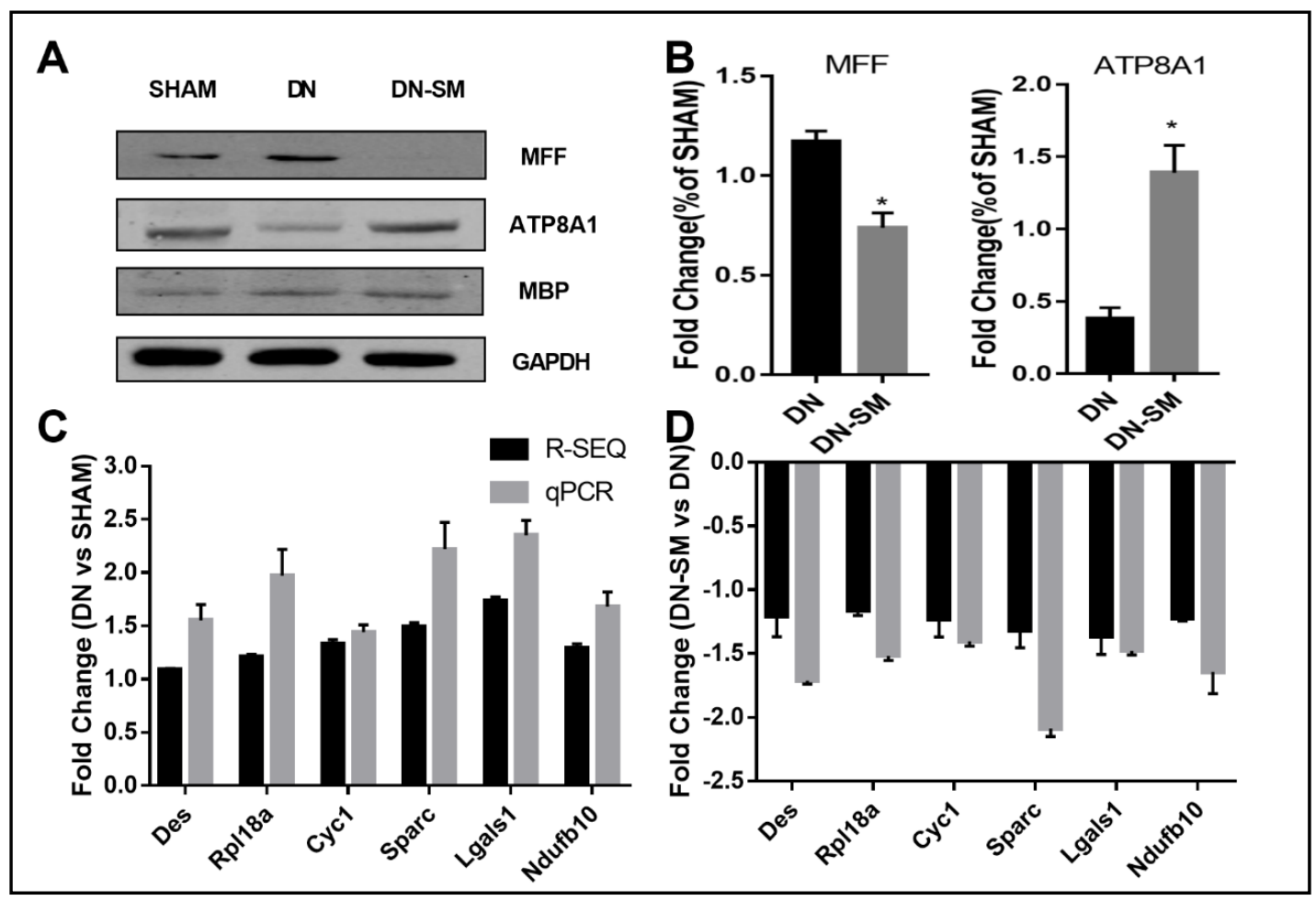

Fig. 5. Western blot and qPCR verification of differentially expressed proteins and genes. (A) Western blot results showing the expression of MFF, ATP8A1, and MBP in the three groups; GAPDH was used as the loading control and MBP was used as a negative control. (B) Statistical analysis of the intensity of the bands (DN-SM vs DN, $n=3$ ). (C and D) qPCR validation of six randomly selected genes from the 402 DEGs, including Des, Rpl18a, Cyc1, Sparc, Lgals1, and Ndufb10; the trends in expression of mRNA detected by qPCR were similar to those detected by RNA-seq.

The sarcomere force is transmitted throughout the cell to the tissue via cytoskeleton proteins. Dystrophin is the most widely studied cytoskeleton protein to date. The dystrophin-associated-glycoprotein (DAG) complex is formed by dystrophin and many associated proteins, and plays a crucial role in maintaining the structural integrity of muscle fibers by linking the extracellular matrix to the subsarcolemmal cytoskeleton, and by providing a scaffold for signaling molecules. Three proteins known to form a complex with DAG were found to be down-regulated after denervation and up-regulated after electrical stimulation. Sarcoglycan beta (SGCB) forms the sarcoglycan sub-complex, which is a protein system playing a key role in sarcolemma stabilization during muscle activity [47]; limb-girdle muscular dystrophy type 2E (LGMD2E) is due to mutations in the SGCB gene $[48,49]$. Sarcoglycan delta (SGCD) is also involved in limb-girdle muscular dystrophy [50]. Dystrobrevin, alpha Dtna-ps1 (DTNA) is a component of the DAG, which is indispensable for the structural integrity of muscle. DTNA also binds to intermediate filaments as well as to syntrophin, a modular adaptor protein considered to be involved in signaling [51]. These three proteins are dystrophin-associated proteins, suggesting an underlying role of DAG in the benefits of the electrical stimulation treatment of denervated muscle.

The final skeletal muscle module that emerged as potentially important in the mechanism of electrical stimulation is the extracellular matrix module. The extracellular matrix surrounds the muscle fibers with multiple layers of organization, and force is transmitted from the sarcomere to the extracellular matrix via the cytoskeleton. The proteins detected in this structure are mainly associated with fibrosis, such as POSTN, COL1A1, COL1A2, COL6A1, COL6A2, COL6A3, FN1, and LUM, which were all up-regulated after denervation and downregulated after electrical stimulation treatment. These results suggest that electrical 


\section{Cellular Physiology Cell Physiol Biochem 2019;52:769-786 \begin{tabular}{ll|l} 
and Biochemistry & $\begin{array}{l}\text { DOl: 10.33594/000000054 } \\
\text { Published online: } 2 \text { April 2019 }\end{array}$ & $\begin{array}{l}\text { O } 2019 \text { The Author(s). Published by } \\
\text { Cell Physiol Biochem Press GmbH\&Co. KG }\end{array}$ \\
\cline { 2 - 3 } &
\end{tabular} \\ La et al.: A Study of Electrical Stimulation on Denervated Muscle}

stimulation might inhibit the fibrosis of denervated skeletal muscle. A previous study also indicated that electrical stimulation could protect against the accumulation of fibrosis in the skeletal muscle of elderly individuals [52]. However, the anti-fibrosis effect of electrical stimulation on denervated muscle has not been widely studied, and is worthy of further consideration.

\section{Conclusion}

In this study, we confirmed that electrical stimulation has a beneficial effect on denervated muscle by maintaining the muscle structure and contractile properties. We further identified 66 differentially expressed proteins and 402 differentially expressed mRNAs that could offer new targets and interesting pathways for in-depth exploration of the underlying mechanism of electrical stimulation on denervated skeletal muscle. In particular, the FoxO and p53 signaling pathways, appear to play a role in these effects and are worthy of further study. The skeletal muscle function modules analysis further revealed a new mechanism for electrical stimulation, including new anti-apoptosis targets, a role in inhibiting muscle fiber type differentiation, protecting against DAG complex mutation, and anti-fibrosis function. Each of these proteins highlighted and their related pathways and modules warrant further detailed consideration to shed new light on the pathogenic mechanisms of muscle denervation as well as the triggers of electrical stimulation.

\section{Acknowledgements}

This project was supported by the National Nature Science Fund of China (11472018). MWZ, GYL, HYX, NL and YYY designed the experiments. GYL, JYL, SO and XXL performed the experiments. GYL and LJZ assessed the data and GYL wrote the manuscript. MWZ, HYX and SO supervised the experiment process, data analysis and the final manuscript written. All authors read and agreed to the final manuscript. This study was approved by the Committee on the Ethics of Animal Experiments of Peking University (permit number: LA201456). All procedures were in strict accordance with the recommendations of the Chinese Laboratory Animal Requirements of Environment and Housing Facilities, and all efforts were made to minimize animals suffering.

\section{Disclosure Statement}

The authors have no conflicts of interest to declare.

\section{References}

- 1 Pellegrino C, Franzini C: An Electron Microscope Study of Denervation Atrophy in Red and White Skeletal Muscle Fibers. J Cell Biol 1963;17:327-349.

2 Gauthier GF, Dunn RA: Ultrastructural and cytochemical features of mammalian skeletal muscle fibres following denervation. J Cell Sci 1973;12:525-547.

3 Heck CS, Davis HL: Effect of denervation and nerve extract on ultrastructure of muscle. Exp Neurol 1988;100:139-153.

4 Sakakima H, Kawamata S, Kai S, Ozawa J, Matsuura N: Effects of short-term denervation and subsequent reinnervation on motor endplates and the soleus muscle in the rat. Arch Histol Cytol 2000;63:495-506.

- 5 al-Amood WS, Lewis DM: A comparison of the effects of denervation on the mechanical properties of rat and guinea-pig skeletal muscle. J Physiol 1989;414:1-16. 


\section{Cellular Physiology Cell Physiol Biochem 2019;52:769-786 \begin{tabular}{ll|l} 
and Biochemistry & $\begin{array}{l}\text { DOl: 10.33594/000000054 } \\
\text { Published online: } 2 \text { April 2019 }\end{array}$ & $\begin{array}{l}\text { O } 2019 \text { The Author(s). Published by } \\
\text { Cell Physiol Biochem Press GmbH\&Co. KG }\end{array}$ \\
\cline { 2 - 3 }
\end{tabular}}

La et al.: A Study of Electrical Stimulation on Denervated Muscle

- 6 Midrio M, Danieli-Betto D, Megighian A, Betto R: Early effects of denervation on sarcoplasmic reticulum properties of slow-twitch rat muscle fibres. Pflugers Arch 1997;434:398-405.

- 7 Albuquerque EX, Thesleff S: A comparative study of membrane properties of innervated and chronically denervated fast and slow skeletal muscles of the rat. Acta Physiol Scand 1968;73:471-480.

8 Ware F, Jr., Bennett AL, Mc IA: Membrane resting potential of denervated mammalian skeletal muscle measured in vivo. Am J Physiol 1954;177:115-118.

9 Dulhunty AF: Excitation-contraction coupling and contractile properties in denervated rat EDL and soleus muscles. J Muscle Res Cell Motil 1985;6:207-225.

- 10 Doucet BM, Lam A, Griffin L: Neuromuscular electrical stimulation for skeletal muscle function. Yale J Biol Med 2012;85:201-215.

11 Su Z, Hu L, Cheng J, Klein JD, Hassounah F, Cai H, Li M, Wang H, Wang XH: Acupuncture plus low-frequency electrical stimulation (Acu-LFES) attenuates denervation-induced muscle atrophy. J Appl Physiol (1985) 2016;120:426-436.

12 Nakagawa K, Tamaki H, Hayao K, Yotani K, Ogita F, Yamamoto N, Onishi H: Electrical Stimulation of Denervated Rat Skeletal Muscle Retards Capillary and Muscle Loss in Early Stages of Disuse Atrophy. Biomed Res Int 2017;2017:5695217.

- 13 Bueno CRS, Pereira M, Favaretto IAJ, Bortoluci CHF, Santos T, Dias DV, Dare LR, Rosa GMJ: Electrical stimulation attenuates morphological alterations and prevents atrophy of the denervated cranial tibial muscle. Einstein (Sao Paulo) 2017;15:71-76.

- 14 Xing H, Zhou M, Assinck P, Liu N: Electrical stimulation influences satellite cell differentiation after sciatic nerve crush injury in rats. Muscle Nerve 2015;51:400-411.

- 15 Lim JY, Han TR: Effect of electromyostimulation on apoptosis-related factors in denervation and reinnervation of rat skeletal muscles. Muscle Nerve 2010;42:422-430.

- 16 Arakawa T, Katada A, Shigyo H, Kishibe K, Adachi M, Nonaka S, Harabuchi Y: Electrical stimulation prevents apoptosis in denervated skeletal muscle. NeuroRehabilitation 2010;27:147-154.

17 Zhang BT, Yeung SS, Liu Y, Wang HH, Wan YM, Ling SK, Zhang HY, Li YH, Yeung EW: The effects of low frequency electrical stimulation on satellite cell activity in rat skeletal muscle during hindlimb suspension. BMC Cell Biol 2010;11:87.

18 Andreose JS, Xu R, Lomo T, Salpeter MM, Fumagalli G: Degradation of two AChR populations at rat neuromuscular junctions: regulation in vivo by electrical stimulation. J Neurosci 1993;13:3433-3438.

19 Kern H, Boncompagni S, Rossini K, Mayr W, Fano G, Zanin ME, Podhorska-Okolow M, Protasi F, Carraro $\mathrm{U}$ : Long-term denervation in humans causes degeneration of both contractile and excitation-contraction coupling apparatus, which is reversible by functional electrical stimulation (FES): a role for myofiber regeneration? J Neuropathol Exp Neurol 2004;63:919-931.

- 20 Bacou F, Rouanet P, Barjot C, Janmot C, Vigneron P, d'Albis A: Expression of myosin isoforms in denervated, cross-reinnervated, and electrically stimulated rabbit muscles. Eur J Biochem 1996;236:539-547.

21 Neilson KA, Ali NA, Muralidharan S, Mirzaei M, Mariani M, Assadourian G, Lee A, van Sluyter SC, Haynes PA: Less label, more free: approaches in label-free quantitative mass spectrometry. Proteomics 2011;11:535553.

22 Lang F, Aravamudhan S, Nolte H, Turk C, Holper S, Muller S, Gunther S, Blaauw B, Braun T, Kruger M: Dynamic changes in the mouse skeletal muscle proteome during denervation-induced atrophy. Dis Model Mech 2017;10:881-896.

23 Sun H, Li M, Gong L, Liu M, Ding F, Gu X: iTRAQ-coupled 2D LC-MS/MS analysis on differentially expressed proteins in denervated tibialis anterior muscle of Rattus norvegicus. Mol Cell Biochem 2012;364:193-207.

24 Cerletti M, Jurga S, Witczak CA, Hirshman MF, Shadrach JL, Goodyear LJ, Wagers AJ: Highly efficient, functional engraftment of skeletal muscle stem cells in dystrophic muscles: Cell 2008;134:37-47.

25 Krivickas LS, Frontera WR: Single muscle fiber physiology in neuromuscular disease. Phys Med Rehabil Clin N Am 2005;16:951-965, ix.

- 26 Ustunel I, Demir R: A histochemical, morphometric and ultrastructural study of gastrocnemius and soleus muscle fiber type composition in male and female rats. Acta Anat (Basel) 1997;158:279-286.

- 27 Frontera WR, Larsson L: Contractile studies of single human skeletal muscle fibers: a comparison of different muscles, permeabilization procedures, and storage techniques. Muscle Nerve 1997;20:948-952. 


\section{Cellular Physiology Cell Physiol Biochem 2019;52:769-786 \begin{tabular}{ll|l} 
and Biochemistry & $\begin{array}{l}\text { DOl: 10.33594/000000054 } \\
\text { Published online: } 2 \text { April 2019 }\end{array}$ & $\begin{array}{l}\text { O } 2019 \text { The Author(s). Published by } \\
\text { Cell Physiol Biochem Press GmbH\&Co. KG }\end{array}$ \\
\cline { 2 - 3 }
\end{tabular}}

La et al.: A Study of Electrical Stimulation on Denervated Muscle

28 Cui H, Liu T, Li P, Yang A, Zhou H, Luo J, Hu E, Hu W, Wang Y, Tang T: An Intersectional Study of LncRNAs and mRNAs Reveals the Potential Therapeutic Targets of Buyang Huanwu Decoction in Experimental Intracerebral Hemorrhage. Cell Physiol Biochem 2018;46:2173-2186.

- 29 Zhong L, Zhou J, Chen X, Liu J, Liu Z, Chen Y, Bai Y: Quantitative proteomics reveals EVA1A-related proteins involved in neuronal differentiation. Proteomics 2017; DOI:10.1002/pmic.201600294.

- 30 Li F, Zhao D, Yang S, Wang J, Liu Q, Jin X, Wang W: ITRAQ-Based Proteomics Analysis of Triptolide On Human A549 Lung Adenocarcinoma Cells. Cell Physiol Biochem 2018;45:917-934.

- 31 Mukund K, Subramaniam S: Co-expression Network Approach Reveals Functional Similarities among Diseases Affecting Human Skeletal Muscle. Front Physiol 2017;8:980.

32 Mukund K, Mathewson M, Minamoto V, Ward SR, Subramaniam S, Lieber RL: Systems analysis of transcriptional data provides insights into muscle's biological response to botulinum toxin. Muscle Nerve 2014;50:744-758.

33 Gregory CM, Bickel CS: Recruitment patterns in human skeletal muscle during electrical stimulation. Phys Ther 2005;85:358-364.

34 Schmalbruch H, al-Amood WS, Lewis DM: Morphology of long-term denervated rat soleus muscle and the effect of chronic electrical stimulation. J Physiol 1991;441:233-241.

- 35 Ashley Z, Salmons S, Boncompagni S, Protasi F, Russold M, Lanmuller H, Mayr W, Sutherland H, Jarvis JC: Effects of chronic electrical stimulation on long-term denervated muscles of the rabbit hind limb. J Muscle Res Cell Motil 2007;28:203-217.

- 36 Salvini TF, Durigan JL, Peviani SM, Russo TL: Effects of electrical stimulation and stretching on the adaptation of denervated skeletal muscle: implications for physical therapy. Rev Bras Fisioter 2012;16:175183.

- 37 Tachtsis B, Smiles WJ, Lane SC, Hawley JA, Camera DM: Acute Endurance Exercise Induces Nuclear p53 Abundance in Human Skeletal Muscle. Front Physiol 2016;7:144.

38 Beyfuss K, Erlich AT, Triolo M, Hood DA: The Role of p53 in Determining Mitochondrial Adaptations to Endurance Training in Skeletal Muscle. Sci Rep 2018;8:14710.

39 Zhang X, Zhang X, Xiong Y, Xu C, Liu X, Lin J, Mu G, Xu S, Liu W: Sarcolemmal ATP-sensitive potassium channel protects cardiac myocytes against lipopolysaccharide-induced apoptosis. Int J Mol Med 2016;38:758-766.

40 Calvo S, Stauffer J, Nakayama M, Buonanno A: Transcriptional control of muscle plasticity: differential regulation of troponin I genes by electrical activity. Dev Genet 1996;19:169-181.

- 41 Ogura Y, Naito H, Kakigi R, Ichinoseki-Sekine N, Kurosaka M, Yoshihara T, Akema T: Effects of ageing and endurance exercise training on alpha-actinin isoforms in rat plantaris muscle. Acta Physiol (Oxf) 2011;202:683-690.

- 42 Frey N, Richardson JA, Olson EN: Calsarcins, a novel family of sarcomeric calcineurin-binding proteins. Proc Natl Acad Sci U S A 2000;97:14632-14637.

- 43 d'Albis A, Goubel F, Couteaux R, Janmot C, Mira JC: The effect of denervation on myosin isoform synthesis in rabbit slow-type and fast-type muscles during terminal differentiation. Denervation induces differentiation into slow-type muscles. Eur J Biochem 1994;223:249-258.

- 44 Bozzo C, Stevens L, Toniolo L, Mounier Y, Reggiani C: Increased phosphorylation of myosin light chain associated with slow-to-fast transition in rat soleus. Am J Physiol Cell Physiol 2003;285:C575-583.

45 Rozanski A, Takano AP, Kato PN, Soares AG, Lellis-Santos C, Campos JC, Ferreira JC, Barreto-Chaves ML, Moriscot AS: M-protein is down-regulated in cardiac hypertrophy driven by thyroid hormone in rats. Mol Endocrinol 2013;27:2055-2065.

46 Pernigo S, Fukuzawa A, Beedle AEM, Holt M, Round A, Pandini A, Garcia-Manyes S, Gautel M, Steiner RA: Binding of Myomesin to Obscurin-Like-1 at the Muscle M-Band Provides a Strategy for Isoform-Specific Mechanical Protection. Structure 2017;25:107-120.

47 Vermiglio G, Runci M, Scibilia A, Biasini F, Cutroneo G: Preliminary study on sarcoglycan sub-complex in rat cerebral and cerebellar cortex. Ital J Anat Embryol 2012;117:54-64.

- 48 Durbeej M, Cohn RD, Hrstka RF, Moore SA, Allamand V, Davidson BL, Williamson RA, Campbell KP: Disruption of the beta-sarcoglycan gene reveals pathogenetic complexity of limb-girdle muscular dystrophy type 2E. Mol Cell 2000;5:141-151. 


\section{Cellular Physiology Cell Physiol Biochem 2019;52:769-786}

\begin{tabular}{cl|l} 
and Biochemistry Published online: 2 April 2019 & $\begin{array}{l}\text { O } 2019 \text { The Author(s). Published by } \\
\text { Cell Physiol Biochem Press GmbH\&Co. KG }\end{array}$ \\
\cline { 2 - 3 }
\end{tabular}

La et al.: A Study of Electrical Stimulation on Denervated Muscle

- 49 Pozsgai ER, Griffin DA, Heller KN, Mendell JR, Rodino-Klapac LR: Systemic AAV-Mediated beta-Sarcoglycan Delivery Targeting Cardiac and Skeletal Muscle Ameliorates Histological and Functional Deficits in LGMD2E Mice. Mol Ther 2017;25:855-869.

- 50 Nigro V, de Sa Moreira E, Piluso G, Vainzof M, Belsito A, Politano L, Puca AA, Passos-Bueno MR, Zatz M: Autosomal recessive limb-girdle muscular dystrophy, LGMD2F, is caused by a mutation in the deltasarcoglycan gene. Nat Genet 1996;14:195-198.

- 51 Nakamori M, Takahashi MP: The role of alpha-dystrobrevin in striated muscle. Int J Mol Sci 2011;12:16601671.

- 52 Kern H, Barberi L, Lofler S, Sbardella S, Burggraf S, Fruhmann H, Carraro U, Mosole S, Sarabon N, Vogelauer M, Mayr W, Krenn M, Cvecka J, Romanello V, Pietrangelo L, Protasi F, Sandri M, Zampieri S, Musaro A: Electrical stimulation counteracts muscle decline in seniors. Front Aging Neurosci 2014;6:189. 